\title{
Fibroscopía Digestiva Alta en Niños Experiencia de 204 Procedimientos
}

Dr. John Wenger K.1.2

\author{
Upper gastro-intestinal tract fiberoptic endoscopy in children.
}

During a 34 montll period, 204 upper gastrointestinal fiberoptic cndoscopies were carried out in 191 pediatric patients General anesthesia was used in two thirds of the cases and the procedure was generally well toleratcd. The youngest patient was 6 weeks old

The procedure identifyed the causal lesions in $26 / 31$ cases of hematemesis and in $8 / 14$ of melena Duodenal ulecr was the most frecuent single cause Icading to upper gastrointestinal bleeding (12 cases). [n $16 / 49$ selected patients with recurrent abdominal pain, a lesion was found at fiberoptic endoscopy; again duodenal ulcer was the commonest cause (6 cases). In patients suspectcd of having esofageal varices upper fiberoptic endoscopy was diagnostic in $8 / 15$, and in those with symptoms suggestive of complications of gastroesofageal roflux a lcsion was found in 17/32.

Additional indications for the procedure were: follow-up of patients with peptic ulcer, endoscopic biopsy in selected celiac patiens. accidental ingestion of caustics and extraction of foreing bodies.

Fiberoptic endoscopy was more succesfull than X-Ray examinations (G.I, series) in identifying the lesions described in this cxperience.

La fibroscopía digestiva alta, es en adultos, un procedimiento altamente difundido y perfectamente incorporado al arsenal diagnóstico gastroen. terológico. En pediatria, la experiencia con este procedimiento es menor y su uso, data solamente desde 1971.(1). Sólo existe una publicación nacional al respecto.(2). De la revisión de casuisticas extranjeras se puede concluir que se trata de una técnica de alto rendimiento llegándose a afirmar $(3,4)$ que "su incorporación a la clínica constituye uno de los avances diagnósticos más importantes de la Gastroenterología Infantil de la última década".

Actualmente la fibroscopía se utiliza como método de diagnóstico y además tiene varias aplicaciones terapéuticas. $(5,6)$

Este examen se puede efectuar en cualquier edad (7), y su utilización no presenta riesgo para el enfermo. Se realiza en pacientes ambulatorios con un simple sedante, pero ocasionalmente se requiere de anestesia general $(3,4,8)$.

Nuestra experiencia con la técnica mencionada

${ }^{1}$ Departamento de Gastroenterología, Hospital L. Calvo Mnckenna.

${ }^{2}$ Trabajo presentado en la Rama de Gastroenterología de la Sociedad Chilena de Pediatria. en el Hospital Luis Calvo Mackenna constituye la base del presente trabajo.

\section{MATERIAL Y METODO}

Desde Diciembre de 1978 hasta Abril de 1982 se efectuaron 204 fibroscopias en 191 niños, cuyas edades fluctuaron entre seis semanas y 17 años. En los de menor edad se prefirió lisar anestesia general, en cambio en escolares y adolescentes muchas veces fue suficiente un sedante suave (Diazepan ß) y un anticolinérgico (Atropina). Ocasionalmente no se utilizó ninguna premedicación (Tabla 1).

Tabla 1

Distribución por Lidad de 204 Fibroscopias

\begin{tabular}{lccc}
\hline & $\begin{array}{c}\text { Con } \\
\text { anestesia } \\
\text { general }\end{array}$ & $\begin{array}{c}\text { Sin } \\
\text { anestesia } \\
\text { gencral }\end{array}$ & Total \\
\hline 1 mes a 1 año & 18 & 0 & 18 \\
1 año a 2 años & 24 & 0 & 24 \\
2 años a 6 años & 43 & 2 & 45 \\
6 an̂os a 10 años & 28 & 14 & 42 \\
10 años a 15 años & 20 & 46 & 66 \\
más de 15 años & 0 & 9 & 9 \\
\hline TOT AL & 133 & 71 & 204 \\
& $(65.1 \%)$ & $(34,8 \%)$ & \\
\hline
\end{tabular}


Los enfermos procedian en su mayoria de la Unidad de Gastroenterología, otros servicios del Hospital L. Calvo Mackenna y ocasionalmente de otros hospitales.

El fibroscopio utilizado es un GIF-P 2 de Olympus de $110 \mathrm{~m}$ de largo, $8.8 \mathrm{~mm}$ de diámetro y de visión frontal de $85^{\circ}$ que se acopla a una fuente de luz modelo CLE 4 U de la misma marca. Este instrumento ha demostrado ser útil en niños mayores de 3 semanas de edad (9).

Se consideraron indicaciones de fibroscopía digestiva las siguientes condiciones clinicas:

1. Hemorragia digestiva alta.

2. Sospecha clinica de esofagitis.

3. Dolor abdominal recurrente con características clinicas de organicidad.

4. Precisar etiología de algunas imágenes radiológicas.

5. Extracción de cuerpo extraño.

6. Ingestión accidental de cáusticos.

La mayor parte de los enfermos tenían un estudio radiológico previo a la fibroscopia. Sólo en los casos de hemorragia digestiva alta este examen se realizó tan pronto como fue posible. Sin embargo algunos fueron referidos tardíamente (hasta 3 meses después del comienzo de los sintomas). Cuando se comprobó la existencia de UHcera péptica, se controló su evolución al completar el tratamiento.

En los casos de dolor abdominal recurrente se utilizó el examen fibroscópico sólo cuando, al finalizar una completa investigación clínica y de laboratorio sin lograr un resultado categórico, persistió la sospecha de una presunta etiología orgánica.

Los pacientes con reflujo gastro-esofágico que presentaron disfagia, anemia o dolor retroesternal en algún momento de su evolución, fueron sometidos a este procedimiento para investigar la existencia de esofagitis. En 25 pacientes consecutivos, portadores de reflujo gastro-esofágico, tratados médicamente según las normas de la Unidad de Gastroenterología y en quienes el examen radiológico mostró desaparición del reflujo, se efectuó fíbroscopía previa al alta para evaluar el aspecto del esófago. En ninguno de esos enfermos se hizo fibroscopia al comienzo del tratamiento.

En los enfermos celíacos, en quienes la biopsia por aspiración con cápsula de Crosby (10) no fue posible, por falta de cooperación del paciente, se tomó muestra de intestino utilizando el fibroscopio con anestesia general. Dicho procedimiento ha demostrado ser útil en adultos con esa patología (11).

\section{RESULTADOS}

En las tablas 2,3,4,5, y 6 se senalan los resultados obtenidos de las 204 endoscopías realizadas y su distribución según la causa que motivódicho examen.
En 49 pacientes con dolor abdominal (Tabla 2) se encontraron 16 lesiones, siendo la más frecuente la Ulcera duodenal. En los casos de esofagitis, gastritis, y duodenitis la fibroscopia demostró ser más eficiente que la radiología para descubrir las lesiones.

Tabia 2

Lndoscopía y Radiología en 49 casos de dolor abdominal Recurrente con sospecha de organicidad.

\begin{tabular}{|c|c|c|c|}
\hline & $\begin{array}{l}\text { Total } \\
\text { Casos } \\
\end{array}$ & $\begin{array}{l}\text { Endos- } \\
\text { copia }\end{array}$ & $\begin{array}{l}\text { Radio- } \\
\text { logia }\end{array}$ \\
\hline Ucera duodenal & 6 & $6(6)$ & $6(6)$ \\
\hline Esofagit is & 3 & $3(3)$ & $0(3)$ \\
\hline Gastritis & 2 & $2(2)$ & $0(2)$ \\
\hline Duodenitis & 5 & $5(5)$ & $1(5)$ \\
\hline Sin lesiones & 33 & $33(49)$ & $42(49)$ \\
\hline Total lesiones & 16 & $16(49)$ & $7(49)$ \\
\hline
\end{tabular}

$($ ) = Número de procedimientos efectuados

Tabla 3

Endoscopía y Radiologia en $\mathbf{4 5}$ casos de hemorragia digestiva.

\begin{tabular}{|c|c|c|c|}
\hline & $\begin{array}{l}\text { Total } \\
\text { Casos }\end{array}$ & $\begin{array}{l}\text { Endox- } \\
\text { copía }\end{array}$ & $\begin{array}{l}\mathbf{R x}^{+} \\
\mathrm{EED}\end{array}$ \\
\hline \multicolumn{4}{|l|}{ Con Hematesis } \\
\hline - Ulcera duodenal & 10 & $10(10)$ & $5(7)$ \\
\hline - Esofagitis & 6 & $6(6)$ & $0(4)$ \\
\hline $\begin{array}{l}\text { - Várices } \\
\text { esolágicos) }\end{array}$ & 4 & $4(4)$ & $1(3)$ \\
\hline $\begin{array}{l}\text { - Ulćera gástrica } \\
\text { - Duodenitis pép- }\end{array}$ & 2 & $2(2)$ & $0(1)$ \\
\hline tica & 2 & $2(2)$ & $0(1)$ \\
\hline $\begin{array}{l}\text { - Gastritis heno- } \\
\text { rrágica }\end{array}$ & 1 & $1(1)$ & $0(0)$ \\
\hline - S. Mallory-Weiss & 1 & $1(1)$ & $0(1)$ \\
\hline Sin lesiones & 5 & $5(31)$ & $11(17)$ \\
\hline Total lesiones & 26 & $\begin{array}{r}26(31) \\
(83.8 \%)\end{array}$ & $\begin{array}{c}6(17) \\
(35,2 \%)^{*}\end{array}$ \\
\hline
\end{tabular}

Sin Hematesis

\begin{tabular}{lllr} 
- Gastritis hemo- & & $2(2)$ & $0(1)$ \\
$\quad$ rrágica & 2 & $2(2)$ & $1(1)$ \\
- Ulcera duodenal & 2 & $2(1)$ & $0(2)$ \\
- Esofagitis & 2 & $2(2)$ & $1(1)$ \\
- Duodenitis & 1 & $1(1)$ & $1(1)$ \\
. Pólipo duodenal & 1 & $1(1)$ & $15(17)$ \\
$\quad$ Sin lesioncs & 6 & $6(14)$ & $2(17)$ \\
\hline Total lesiones & 8 & $8(14)$ & $(11,7 \%)^{*}$ \\
\hline
\end{tabular}

+ Radiografías de esófago, estómago y duodeno.

() Nímero de procedimientos efectuados.

* Proporción de casos en que cada procedimiento fue positivo. 
Los pacientes con hemorragia digestiva se presentan en dos grupos, dependiendo de la presencia de hematemesis. (Tabla 3). En el grupo de enfermos que tuvieron hematemesis se encontró la causa del sangramiento en 26 de $31(83,8 \%)$, siendo la úlcera duodenal el origen más frecuente. En los enfermos con melena sin hematemesis, se encontraron lesiones en 8 de 14 casos $(57,1 \%)$. En uno de ellos se demostró posteriormente la existencia de un divertículo de Meckel.

Aunque frecuentemente los resultados radiológicos y fibroscópicos concordaron, ocasionalmente la fibroscopía demostró una etiologia diferente y a veces no sospechada por la clinica. Así por ejemplo, en 2 lactantes con hemorragia digestiva sin elementos clínicos de hipertensión portal, se encontraron várices esofágicas que posteriormente se confirmaron con la esplenoportografía. En otro lactante con hematemesis se encontró una intensa esofagitis moniliásica sin que hubiera una infección oral por el mismo agente. En un escolar en que el estudio radiológico sugirió la existencia de un gran nicho en el bulbo duodenal, la fibroscopia demostró que esa imagen radiológica correspondía a un divertículo con mucosa duodenal normal.

La sospecha de hipertensión portal condujo a la exploración de 15 enfermos, con el objeto de detectar la existencia de várices esofágicas (Tabla 4), las que se encontraron en 8 casos. La búsqueda radiológica en 10 de estos pacientes, sólo demostró várices en 2 . En un paciente de este grupo, en quien clínicamente se sospechó la existencia de várices esofágicas, éstas no se confirmaron y en cambio se encontró esofagitis severa asociada a reflujo gastro-esofágico evidente radiológicamente. Este paciente nunca habia sido vomitador.

\section{Tabla 4.}

Endoscopia y Radiologia en pacientes con sospecha de hipertensión portal. Búsqueda de várices escofágicas.

\begin{tabular}{lccl}
\hline & $\begin{array}{l}\text { Total } \\
\text { casos }\end{array}$ & $\begin{array}{l}\text { Endos- } \\
\text { copía }\end{array}$ & $\begin{array}{l}\text { Radio- } \\
\text { logía }\end{array}$ \\
\hline Con várices & 8 & $8(8)$ & $2(6)$ \\
Sin várices & 7 & $7(7)$ & $4(4)$ \\
\hline Total casos & & & \\
positivos & 8 & $8(15)$ & $2(10)$ \\
\hline
\end{tabular}

( ) Número de procedimientos efectuados.

En el grupo de enfermos con reflujo gastro-esofágico con sospecha de complicación, se encontró que la lesión más frecuente es la esofagitis y que la fibroscopia tiene mucho más rendimiento que la radiología en su diagnóstico. La existencia de estenosis asociada es mucho menos frecuente. (Tabla 5).

\section{Tabla 5}

Éndoscopia y Radiología efectuados en 32 casos de reflujo gastro-esofágico con sospecha de complicación.

\begin{tabular}{lccc}
\hline & $\begin{array}{c}\text { Total } \\
\text { casos }\end{array}$ & $\begin{array}{l}\text { Endos- } \\
\text { copía }\end{array}$ & $\begin{array}{c}\text { Radio- } \\
\operatorname{logía}\end{array}$ \\
\hline $\begin{array}{l}\text { Esofagitis } \\
\text { Esofagitis } y \\
\text { estenosis } \\
\text { Grastritis hemo- }\end{array}$ & 9 & $9(9)$ & $2(7)$ \\
$\begin{array}{l}\text { rágica } \\
\text { Sin lesión }\end{array}$ & 3 & $5(5)$ & $4(4)$ \\
Total lesiones & 15 & $15(32)$ & $22(28)$ \\
\hline
\end{tabular}

( ) Número de procedimientos efectuados.

En los 25 enfermos sometidos a fibroscopía al finalizar con éxito el tratamiento médico de reflujo gastroesofágico, se encontró total indemnidad endoscópica en todos los casos (Tabla 6).

\section{Tabla 6}

\section{Otros Enfermos sometidos a Endoscopia}

Reflujo gastro-esofágico mejorado

con tratamiento médico ..........25 casos

Ulcera péptica

tratada (control evolución); , . . . . . . . 12

Ulcera cicatrizada .9 casos
Ulcera activa $\ldots .2$
Duodenitis ... 1

Biopsia endoscópica de duodeno en pacientes celíacos . . . . . . . . . 21

Ingestion accidental de cautıcos $\ldots \ldots \ldots \ldots 2$

Extracción de cuerpo extraño. . . . . . . . . 1

Esofagitis traumática por cuerpo extraño . . 1

Angiomas múltiples en esófago . . . . . . . 1

TOTAL $\ldots \ldots \ldots \ldots \ldots 63$

De los 20 pacientes con úlcera péptica, 12 fueron controlados endoscópicamente al final del tratamiento médico. Se demostró regresión de la lesión en 9 de ellos, persistencja de la úlcera activa en dos y duodenitis sin úlcera en uno.

En los 21 enfermos celíacos sometidos a endoscopia alta para biopsia duodenal se obtuvieron muestras adecuadas para fines diagnósticos, aunque tendieron a ser más pequeñas que con el sistema convencional. 
Las dos endoscopias indicadas por ingestión de cáustico, correspondieron al mismo enfermo, que tenía una esofagitis grave por ingestiôn accidentàl de ácido sulfúrico.

El paciente de cinco meses al que se extrajo un alfiler de gancho enclavado en el duodeno, fue comunicado en una publicación anterior (12).

Ia esofagitis traumática correspondió a un escolar con intensa disfagia, provocada al tragar accidentalmente un palito de caramelo.

Los angiomas múltiples del esófago fueron encontrados en un lactante cuyo estudio radiológico previo sugirió la existencia de tales lesiones.

En las 204 endoscopias se produjeron dos complicaciones atribuibles al procedimiento. Un lactante menor con anestesia general presentó un paro cardiaco reversible a los pacos segundos. Utro enfermo toleró bien el procedimiento pero tuvo hiperemcsis 12 horas después necesitando hidratación parenteral. En este enfermo no se encontró elevación de los valores normales de amilasemia ni amilasuria.

\section{DISCUSION}

En el $65,1 \%$ de nuestras endoscopias se utjlizó anestesia general. Ament to hizo en el $73 \%$ (13). Liebman en el $62 \%(7)$, y Goldstein Gleason y Tedesco no la utilizaron $(14,15,16)$. En nuestra experiencia, la anestesia general ha demostrado ser un método que facilita un examen endoscópico minucioso y es insustituible en los pacientes que no cooperan, sobre todo en los más pequeños.

En los niños con dolor abdominal recurrente los factores emocionales son responsables de los síntomas en la mayoría de los casos (17), sólo una pequeña proporción tiene causa orgánica que lo explique. De los pacientes escogidos por nosotros (en base a los elementos clinicos que nos sugirieron una presunta etiologia orgánica) el $32,6 \%$ de los casos demostraron tenet una lesión, to que representa una proporción similar a la encontrada por Ament (4, 13).

En el $83,8 \%$ de los niños con hematemesis se identifico la causa del sangramiento. Ament la encuentra en el $80 \%$ (4). Se sabe que una demora de 24 a 48 horas en la exploración endoscópica disminuye considerablemente su rendimiento (18 19). Es probable que algunos enfermos con melena, sin hematemesis correspondan a sangramientos bajo el ángulo de Treitz, inaccesibles al fibroscopio.

En numerosas publicaciones se ha establecido que el rendimiento del examen fibroscópico es superior al radiológico en los casos de hemorragia digestiva alta $(4,8,15,16,19,20,2122,23,24$. 25). Este hecho es explicable por cuanto ciertas lesiones superficiales como esofagitis, gastritis, duodenitis. pueden pasar inadvertidas al examen con bario $y$ sólo son identificadas con visión directa endoscópica. Sin embargo, debe recordarse que la Radiología y la Endoscopía son procedimientos complementarios y no excluyentes, ciertos aspectos de la motilidad del tubo digestivo y la búsqueda de lesiones más allá del ligamento de Treitz. requieren del empleo de Rayos X.

En los enfermos con patología esofágica, es de interés comprobar que los tratados médicamente por reflujo gastro-esofágico sin síntomas, mejoran con un esófago totalmente sano endoscópicamente. En cambio, los enfermos con reflujo que presentaron síntomas de cierta importancia (disfagia, dolor retroesternal, anemia) tuvieron lesión endoscópicamente identificable en el $53,1 \%$ de los casos. Los pacientes de este grupo no habian recibido tratamiento médico, o lo estaban recibiendo en forma defectuosa.

En los pacientes con várices esofágicas también se demostró un mayor rendimiento del examen endoscópico sobre el radiológico, experiencia coincidente con la de otros grupos $(21,28)$. Debe recordarse además que la radiologia puede llevar a falsas interpsetaciones en presencia de pólipos esofágicos: que sólo pueden diferenciarse de las várices esofágicas mediante la endoscopílas (26).

El uso del fibroscopio para obtener biopsias duodenales en los portadores de Enfermedad Celíaca es de excepción y sólo está inđicado cuando no es posible usar el método convencional. Las pinzas de los fórceps de los fibroscopios permiten obtener muestras adecuadas pero más pequefias y superficiales que la cápsula de succión. Recientemente se ha comunicado una técnica combinada de cápsula de succión conectada a una sonda que se introduce a través del fibroscopio (27).

La morbilidad atribuible a la fibroscopía digestiva alta es escasa. En nuestros enfermos sólo tuvimos 2 casos $(0,9 \%)$. Ament describió erosiones gástricas en enfermos mal tranquilizados, depresión respiratoria en pacientes excesivamente medicados e hiperemesis con amilasemia y amilasuria elevadas en casos de introducción del endoscopio en la Papila de Vater $(3,4)$. La evolución de los enfermos por el descritos, asi como también los de nuestra serie, ha sido satisfactoria en todos los casos.

\section{RESUMEN}

Se presenta la experiencia en 204 endoscopias digestivas altas realizadas a niños mayores de 6 semanas en el Hospital L. Calvo Mackenna entre 1978 y 1982. E1 65,1\% de las intervenciones se efectuó con anestesia general.

El procedimiento permitió encontrar 16 lesiones en 49 enfermos con dolor abdominal recurrente sugerente de organicidad, 34 lesiones en 45 enfermos con hemorragia digestiva, 8 casos de 
várices esofágicas en 15 enfermos con sospecha de hipertensión portal y 17 lesiones en 32 enfermos con reflujo gastro-esofágico con signos de complicación. Este procedimiento también se utilizó para controlat la evolución de los enfermos con úlcera péptica $y$ en forma excepcional en algunos enfermos celíacos para obțener biopsia de intestino delgado.

En algunos casos la fibroscopía tiene claras ventajas sobre el examen radjológico de tubo digestivo, sin embargo ambos procedimientos no son excluyentes sino complementatios.

Se agradece al Servicio de Anestesia del Hospital L. Calvo Mackenna la colaboración prestada, ya que sin ella este trabajo habría sido imposible.

\section{REFERENCIAS}

${ }^{1}$ Gans S.L. y Berci G.: "Advances in endoscopy of infants and chilaren". J. Pediatr. Surg. 6: 199, 1971.

${ }^{2}$ Rudloff $T$., Cabrera M. Riel $F$, y Col."Panendoscopia digestiva alta en el niño" Rev. Chil. Pediat. 52: 289, 1981

${ }^{3}$ Ament M.E. y Christie D.L.:"Upper gastrointestina? fiberoptic endoscopy in pediatric patients". Gastroenterol. $72: 1244,1977$.

4 Ament M.E.: "Upper gastrointestinal fiberoptic endoscopy in pediatric patients Experience with 209 procedures in 180 patients". En Lebenthal, E. (ed). "Digestive Disease in children " firume \& Stratton, New York 1978) pp $3: 19$.

5 Christie D.L. y Ameni M.E.."Rennoval of foreignbodies from esofagus and stomach with Mexible fiberoptic panendoscope". Pediatr. 57: 9311956

6 Liebman W.M.:"Fiberoptic endoscopy of the gastrointestinal tract in infants and children II - Fiberoptic colonoscopy and polipectomy in 15 children". Am J. Gastroenterol. 68: 452, 1977.

${ }^{7}$ Liebman 'W.M., Thaler M. M. y Bujanover T.: "Endoscopic evaluation of upper gastrointestinal bleeding in the newborn".A m. J. Gastroenterol. 69:607. 1978.

"Liebman W.M.:*"Fibereptic endoscopy of the gastrointestinal tract in infants and children. l-Upper endoscopy in 53 children'. Am. J. Gastroenterol 68; $362,1977$.

9 Ament M.E.:"A new prototype 1-channel. 4- way tip control pediatric upper gastrointestinal fibercope". Gastrointest. Endosc. 23: 139, 1977.

10 Guiraldes $E$, Gutierrez $C$ y Latorre J.J.".Biopsia intestinal en Enfermedad Celíaca". Rev. Mod. Ch. 105: $553,1977$.

11 Gillberg $R$. $y$ Ahren $C .{ }^{t}$ Celiac Disease diagnosed by means of duodenoscopy and endoscopic duodenal biopsy". Scand. J. Gastroent. 12: 911, 1977.
12 Wenger J. y Lira E.:"Extracción de un cuerpo extraño del duadeno con un tibroscopio". Rev. Ch. Pediat. 51: $445,1980$.

13 Ament M.E., Gans S.L. y Christie D.L.:"Experience with Esofagogastroduodenoscopy in diagnosis of 79 pediatric patients with hematemesis, melena or chronic abdominal pain : Gastroenterol, 68: 858, 1975.

${ }^{14}$ Goldstein P.D., Tedesco F.J, y Gleason W.A.:"Gastrointestinal fiberendoscopy as a diagnostic tool in infants and children". (Abstr.). Gastroenterol. 68: 997, 1975.

${ }^{15}$ Gleason W.A., Tedesco F.J., Keuting J.P. et al."Fiberoptic gastrointestinal endoscopy in infants and children". J. Pedíat. 85: 810, 11974.

16 Tedesco F.L., Goldstein P.D., Glenson W.A. et al: 'Upper gastrointestinal endoscopy in pediatric patients". Gastroenterol. 70: 492, 1976.

${ }^{17}$ Roy C.C., Silvermenn A. y Cozzetto F.J.:"Psychophysiologic recurrent abdominal pain". En "Pediatric Clinical Gastroenteroloy". Second Ed. pp342. (The C.V. Mosby Company). 1975.

18 Leinicke J.A., Shafer R.D., Hogan W.J. et al: "Emergency endoscopy in acute gastrointestinal bleeding: Does timing affect the significance of diagnostic yield?". Gastrointest. Endosc. 22: 228, 1976.

19 Mitchell C.J. y Jewell D.P.:" The diagnosis of the site of upper gastrointestinal hemorthage in patients with established portal hypertension". Endoscopy 9: 131, 1977.

${ }^{20}$ Cox K. y A ment M.E.+-"Upper gastrointestinal bleeding in children and adolescents". Pediatr. 63: 408, 1979.

21 Liebman W.M.:" Diagnosis and management of upper gastrointestinal hemorrhage in children". Pediatr. Ann 5: 690, 1976.

22 Gilbert D.A., Silverstein D.E., Tedesco F.J. et al: "Prognosis of upper gastrointestinal bleeding. Preliminary results of the ASGE National bleeding survey". Gastroenterol. 76: I138, 1979.

${ }^{23}$ Cotton P.B.. Rosenberg M.T., Waldram R.P. L et al:"Early endoscopy of esofagus, stomach and duodenal bulb in patients with hematemesis and melena" $\mathrm{Br}$. Med. J. 2: 505, 1973.

24 Stevenson G.W., Cox R.R. y Roberts C.J.:"Prospective comparison of double contrast barium meal examination and fiberoptic endoscopy in acuite upper gastrointestinal hemorrhage". Br. Med. J. 2: 723, 1976.

${ }^{25}$ Schiller K.F.R. y Cotton P.B.:" Acute Upper Gastrointestinal hemorrhage". Clin. Gastroenterol. 7: 595, 1978.

26 Branski D., Gardner R.U., Fisher J.E. et al:"Gastroesofageal polip as a cause of hematemesis in adolescence". Am. J. Gastroenterol. 73: 448, 1980.

27 Holdstock $G_{4}$ " Jejunal biopsy without the need for screening". Lancet, I: $1236,1978$.

20 Cadranel S., Rodesch P., Peeters I.P. et al: "Fiberendoscopy of the gastrointestinal tract in Children". Am. J. Dis. Child. 131: 41, 1977. 